7th International Workshop on Astronomy and

Relativistic Astrophysics (IWARA 2016)

International Journal of Modern Physics: Conference Series

Vol. 45 (2017) 1760034 (5 pages)

(C) The Author(s)

DOI: $10.1142 / \mathrm{S} 2010194517600345$

\title{
Delta Resonance Coupling with Walecka's Mesons: Implications to Stellar Matter EoS
}

\author{
William Silva Gomes, José Carlos Teixeira de Oliveira \\ Universidade Federal de Roraima (UFRR) \\ Campus do Paricarana s/n, Boa Vista, Roraima, 69310-270, Brazil \\ willfis01@gmail.com, jose.oliveira@ufrr.br \\ Hilário Rodrigues \\ Centro Federal de Educação Tecnológica Celso Suckow da Fonseca (CEFET-RJ) \\ Av. Maracanã, 229, Rio de Janeiro, RJ, 20271-110, Brazil \\ harg@cefet-rj.br \\ Sérgio B. Duarte \\ Centro Brasileiro de Pesquisas Físicas (CBPF) \\ Rua Dr. Xavier Sigaud, 150, Rio de Janeiro, RJ, 22290-180, Brazil \\ $s b d @ c b p f . b r$
}

Published 15 August 2017

In this work we have obtained the equation of state to the highly asymmetric dense stellar matter, using the nonlinear Walecka model in the mean field approximation. We discussed the implication of changes in coupling constant of the delta baryonic resonance on the observable of the neutron star. A detailed analysis of the equation of state and of the baryonic effective mass in respect to changes in the delta coupling constants is carried out. We focus attention on a new aspect observed for pressure when varying the baryonic density of the medium; a first order phase transition like a liquid-gas phase transition was observed for an acceptable range of delta coupled constant values. We have explored the implication of this aspect for the neutron star structure and their maximum masses.

Keywords: Stars, Delta Resonances, Nonlinear Walecka Model, Equation of State.

PACS numbers: 21.65.Mn; 26.60.Kp

\section{Introduction}

The high production of delta-resonances in the dense phase $\left(\rho \geq 3 \rho_{0}\right)$ of relativistic heavy ion collisions ${ }^{1,2}$, where $\rho_{0}$ is the normal nuclear matter density, leads to a

This is an Open Access article published by World Scientific Publishing Company. It is distributed under the terms of the Creative Commons Attribution 4.0 (CC-BY) License. Further distribution of this work is permitted, provided the original work is properly cited. 
great interest in the study on the delta matter effect to the neutron star structure. In this work we obtained the equation of state of baryons and leptons to the highly asymmetric dense stellar matter. We discussed the implication of changes in coupling constant of the delta baryonic resonance with Walecka's mesons to the formation of delta resonance matter in stellar medium. We adopt the nonlinear Walecka model in the mean field approximation ${ }^{3,4}$, including the octet of baryons with spin $1 / 2(n$, $\left.p, \Lambda^{0}, \Sigma^{-}, \Sigma^{0}, \Sigma^{+}, \Xi^{-}, \Xi^{0}\right)$, the baryonic resonances of spin $3 / 2$ with isospin states $\left(\Delta^{-}, \Delta^{0}, \Delta^{+}, \Delta^{++}\right)$and $\Omega^{-}$resonance in the baryonic sector ${ }^{5,6}$. In the leptonic sector we consider the electrons and muons.

\section{The Lagrangian Density}

The Lagrangian density of this hadronic and leptonic dense medium ${ }^{3,4}$ is given by

$$
\mathscr{L}=\mathscr{L}_{\mathrm{F}}+\mathscr{L}_{\mathrm{I}}
$$

where

$$
\begin{aligned}
\mathscr{L}_{\mathrm{F}}= & \sum_{B} \bar{\Psi}_{B}\left(i \gamma_{\mu} \partial^{\mu}-m_{B}\right) \Psi_{B}+\sum_{\zeta=\Delta, \omega} \bar{R}_{\zeta \nu}\left(i \gamma_{\mu} \partial^{\mu}-m_{\zeta}\right) R_{\zeta}^{\nu} \\
& +\frac{1}{2}\left(\partial_{\mu} \sigma \partial^{\mu} \sigma-m_{\sigma}^{2} \sigma^{2}\right)-\frac{1}{3} b m_{N}\left(g_{\sigma_{N}} \sigma\right)^{3}-\frac{1}{4} c\left(g_{\sigma_{N}} \sigma\right)^{4}-\frac{1}{4} \omega_{\mu \nu} \omega^{\mu \nu} \\
& +\frac{1}{2} m_{\omega}^{2} \omega_{\mu} \omega^{\mu}-\frac{1}{4} \rho_{\mu \nu} \rho^{\mu \nu}+\frac{1}{2} m_{\rho}^{2} \rho_{\mu} \rho^{\mu}+\sum_{\lambda=e^{-}, \mu^{-}} \bar{\Psi}_{\lambda}\left(i \gamma_{\mu} \partial^{\mu}-m_{\lambda}\right) \Psi_{\lambda},
\end{aligned}
$$

is the Lagrangian density for free baryons, free leptons $\left(e^{-}, \mu^{-}\right)$, and the meson fields $\sigma, \omega_{\mu}$ and $\rho_{\mu}$. In addition, the interaction Lagrangian density is given by the following expression

$$
\begin{aligned}
\mathscr{L}_{\mathrm{I}}= & \sum_{B} \bar{\Psi}_{B}\left(g_{\sigma_{B}} \sigma-g_{\omega_{B}} \gamma_{\mu} \omega^{\mu}-\frac{1}{2} g_{\rho_{B}} \gamma_{\mu} \tau \rho^{\mu}\right) \Psi_{B}+ \\
& \sum_{\zeta=\Delta, \Omega} \bar{R}_{\zeta \nu}\left(g_{\sigma \zeta} \sigma-g_{\omega \zeta} \gamma_{\mu} \omega^{\mu}-\frac{1}{2} g_{\rho \zeta} \gamma_{\mu} \tau \rho^{\mu}\right) R_{\zeta}^{\nu} .
\end{aligned}
$$

In the equations above the operator $\Psi_{B}$ represents the Dirac spinor describing the 1/2-spin baryonic octet, with $B=n, p, \Lambda^{0}, \Sigma^{-}, \Sigma^{0}, \Sigma^{+}, \Xi^{-}, \Xi^{0}$ and the operator $R_{\zeta}^{\nu}$ is the Rarita-Schwinger spinor ${ }^{7}$, with $\zeta=\Delta^{-}, \Delta^{0}, \Delta^{+}, \Delta^{++}, \Omega^{-}$describing the 3/2-spin baryonic resonances.

\section{Results and Conclusions}

In Figs. 1(a) and (b) we show the behaviour of the equation of state (pressure versus density) for different values of delta-mesons coupling constants. Notice in figure 1 (a), the presence of some intervals for which $d P / d \rho<0$, that indicates the existence of a phase transition due to the production of delta-resonance in the medium. We note that the attractive nature of the delta resonance interaction with other baryons 
induces a first order phase transition like a liquid-gas phase transition. A Maxwell construction is thus implemented in order to connect both the phases. In Fig. 1(b) we show the behaviour of the pressure versus density after the Maxwell construction.
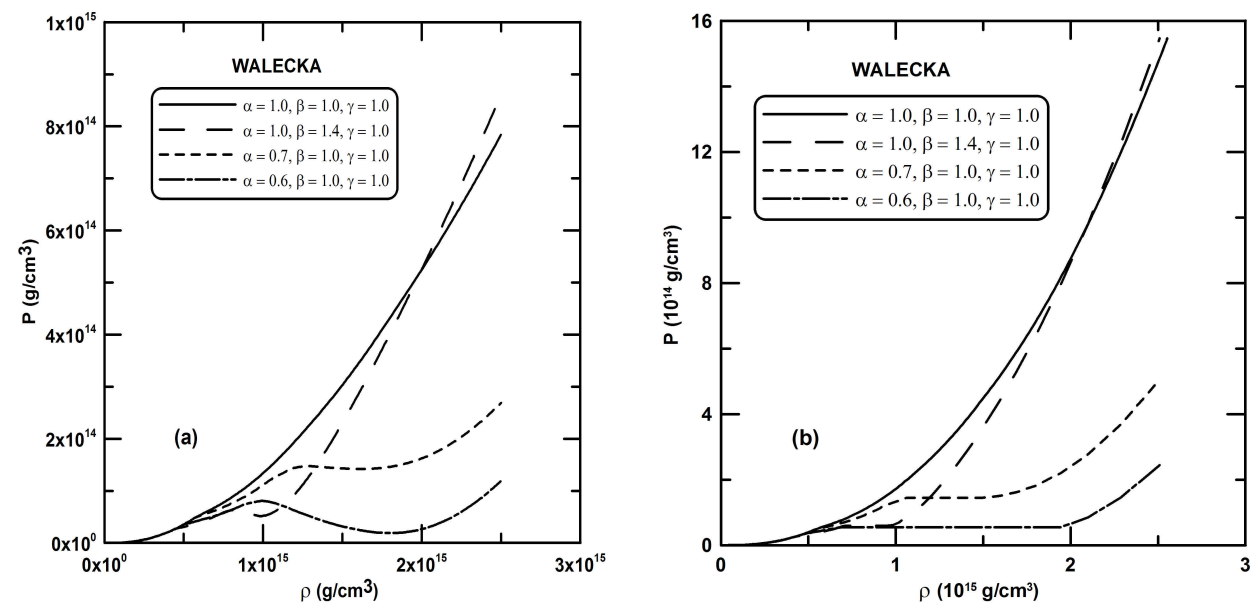

Fig. 1. Pressure as a function of density for different values of delta-mesons coupling constants, in (a), and the pressure as a function of density after the Maxwell construction, in (b).

In this work we have used the results of the finite density QCD Sum Rules (QCDSR) to determine the possible values of delta-meson coupling constants ${ }^{8}$, where we have taken different values for the quantities $\alpha=g_{\omega \Delta} / g_{\omega N}, \beta=g_{\sigma \Delta} / g_{\sigma N}$ and $\gamma=g_{\rho \Delta} / g_{\rho N}$. For the nonlinear Walecka model we have used the set of coupling constants given in Ref. 9, namely $\left(g_{\sigma N} / m_{\sigma}\right)^{2}=9.927 \mathrm{fm}^{2},\left(g_{\omega N} / \mathrm{m}_{\omega}\right)^{2}=4.820$ $\mathrm{fm}^{2},\left(g_{\rho N} / \mathrm{m}_{\rho}\right)^{2}=4.791 \mathrm{fm}^{2}, b=0.008659$ and $c=-0.002421$.

In figure 2 we show the behaviour of the effective mass for different values of delta-mesons coupling constants. We observe that the effective mass is reduced for the cases: $(\alpha=1.0, \beta=1.4, \gamma=1.0),(\alpha=0.7, \beta=1.0, \gamma=1.0)$ and $(\alpha=0.6$, $\beta=1.0, \gamma=1.0)$, when compared to the case universal coupling $(\alpha=\beta=\gamma=1.0)$.

Using the equation of state obtained by the model, we have numerically solved the Tolman-Oppenheimer-Volkoff (TOV) structure equations ${ }^{10,11}$ in order to obtain the mass-radius relationship of a neutron star. The figure 3 shows the mass-radius diagrams for the sets of parameters: $(\alpha=\beta=\gamma=1.0),(\alpha=1.0, \beta=1.4, \gamma=1.0)$, $(\alpha=0.7, \beta=1.0, \gamma=1.0)$ and $(\alpha=0.6, \beta=1.0, \gamma=1.0)$.

\section{Conclusion}

In conclusion, we remark that the neutron star has a maximum mass when we use the parameter $(\alpha=\beta=\gamma=1.0)$. For this case, where the delta resonances appear near $\rho=8 \rho_{0}$, the equation of state is stiffer, and thus this explains why the neutron star can support more matter. 
W. S. Gomes et al.

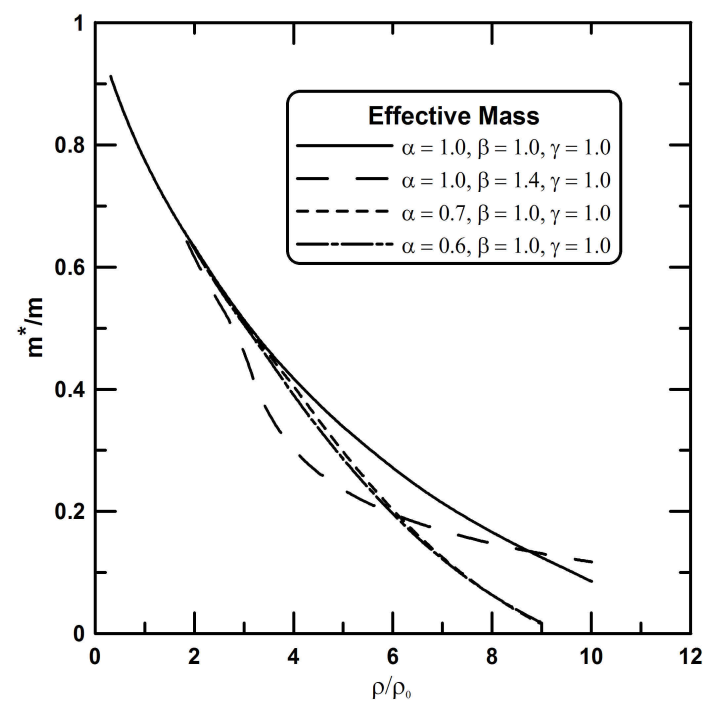

Fig. 2. Effective mass versus density for different values of delta-mesons coupling constants.

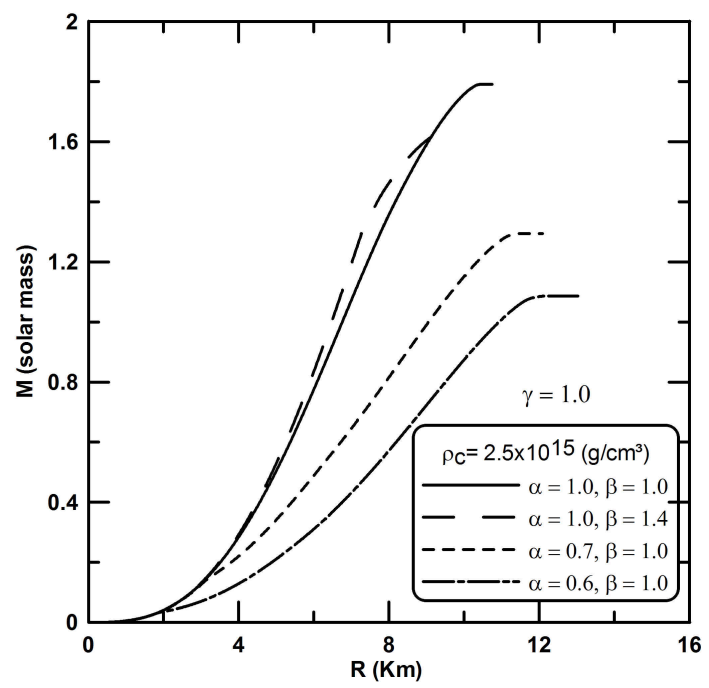

Fig. 3. Mass-radius diagrams for different values of delta-mesons coupling constants, with central density $\rho_{c}=2.5 \times 10^{15} \mathrm{~g} / \mathrm{cm}^{3}$.

\section{References}

1. E. L. Hjort et al., Phys. Rev. Lett. 79, 4345 (1997).

2. M. Hofmann et al., Phys. Rev. C 51, 2095 (1995).

3. J. D. Walecka, Ann. Phys. 83, 497 (1974).

4. B. D. Serot and J. D. Walecka, Adv. Nucl. Phys. 16, 1 (1986).

5. J. C. T. Oliveira et al., Mod. Phys. Lett. A 15, 1529 (2000). 
6. J. C. T. Oliveira et al., Int. J. Mod. Phys. D 16, 175 (2007).

7. W. Rarita and J. Schwinger, Phys. Rev. 60, 61 (1941).

8. D. S. Kosov et al., Phys. Lett. B 37, 421 (1998).

9. N. K. Glendenning and S. A. Moszkowski, Phys. Rev. Lett. 67, 2414 (1991).

10. R. C. Tolman, Phys. Rev. 55, 364 (1939).

11. J. R. Oppenheimer and G. M. Volkoff, Phys. Rev. 55, 374 (1939). 